\title{
Optimal Power Allocation in Multiuser OFDM Systems
}

\author{
Zukang Shen, Jeffrey G. Andrews, and Brian L. Evans \\ Wireless Networking and Communications Group \\ Department of Electrical and Computer Engineering \\ The University of Texas at Austin, Austin, Texas 78712 \\ Email: $\{$ shen, jandrews, bevans\}@ece.utexas.edu
}

\begin{abstract}
Multiuser orthogonal frequency division multiplexing (MU-OFDM) is a promising technique for achieving high downlink capacities in future cellular systems. A key issue in MU-OFDM is the allocation of the OFDM subcarriers and power among users sharing the channel. Previous allocation algorithms cannot ensure fairness in advance. In this paper, a proportional rate adaptive resource allocation method for MUOFDM is proposed. Subcarrier and power allocation are carried out sequentially to reduce the complexity, and an optimal power allocation procedure is derived, through which proportional fairness is achieved. Simulation results show that this lowcomplexity MU-OFDM system achieves double the capacity of a fixed time division approach to OFDM multiple access, and also has higher capacity than previously derived suboptimal power distribution schemes.
\end{abstract}

\section{INTRODUCTION}

Orthogonal frequency division multiplexing (OFDM) is a promising technique for the next generation of wireless communication systems [1] [2]. OFDM divides the entire transmission bandwidth into $N$ orthogonal subchannels. By adding a cyclic prefix (CP) to each OFDM symbol, the channel appears to be circular if the $\mathrm{CP}$ length is longer than the channel length, in which case both intersymbol and intercarrier interference can be removed. Each subchannel thus can be modeled as a gain plus additive white Gaussian noise (AWGN). Besides the improved immunity to fast fading [3] brought by the multicarrier property of OFDM systems, multiple access is also possible because the subchannels are independent of each other.

Multiuser OFDM adds multiple access to OFDM by allowing a number of users to share an OFDM symbol. Two classes of resource allocation schemes exist: fixed resource allocation [4] and dynamic resource allocation [5] [7] [8]. Fixed resource allocation schemes, such as time division multiple access (TDMA) and frequency division multiple access (FDMA), assign an independent dimension, e.g. time slot or subchannel, to each user. A fixed resource allocation scheme is not optimal because the scheme is fixed regardless of the current channel condition. On the other hand, dynamic resource allocation allocates a dimension adaptively to the users based on their channel gains. Due to the time-varying nature of the wireless

B. L. Evans was supported by The State of Texas Advanced Technology Program under project 003658-0614-2001. channel, dynamic resource allocation makes full use of the multiuser diversity to achieve higher performance.

Two classes of optimization techniques have been proposed in the dynamic multiuser OFDM literature: margin adaptive (MA) [5] and rate adaptive (RA) [6], [7]. The margin adaptive objective is to achieve the minimum overall transmit power given the constraints on the users' data rate or bit error rate (BER). The rate adaptive objective is to maximize each user's error-free capacity with a total transmit power constraint. These optimization problems are nonlinear and hence computationally intensive to solve. In [8], the nonlinear optimization problems were transformed into a linear optimization problem with integer variables. The optimal solution can be achieved by integer programming. However, even with integer programming, the complexity increases exponentially with the number of constraints and variables.

In this paper, a proportional rate adaptive optimization is proposed. Instead of trying to maximize the minimum user's capacity as in [7], the proposed RA optimization tries to maximize the overall user's capacity while maintaining proportional fairness among users. Furthermore, since it is typically very hard to optimize the subcarriers and power jointly, these two procedures are carried out separately to lower the complexity.

This paper is organized as follows. Section II introduces the multiuser OFDM system model and presents the optimization objective function. In Section III, the optimal multiuser power allocation scheme is developed. Simulation results are presented in Section IV. Conclusions are drawn in Section V.

\section{SYSTEM MODEL}

Fig. 1 depicts a multiuser OFDM system. In the base station, all channel information is sent to the subcarrier and power allocation algorithm through feedback channels from all mobile users. The resource allocation scheme made by the algorithm is forwarded to the OFDM transmitter. The transmitter then selects different numbers of bits from different users to form an OFDM symbol. The resource allocation scheme is updated as fast as the channel information is collected. In this paper, perfect instantaneous channel information is assumed to be available at the base station and only the broadcast scenario is studied. It is also assumed that the subchannel and bit allocation information are sent to each user by a separate 


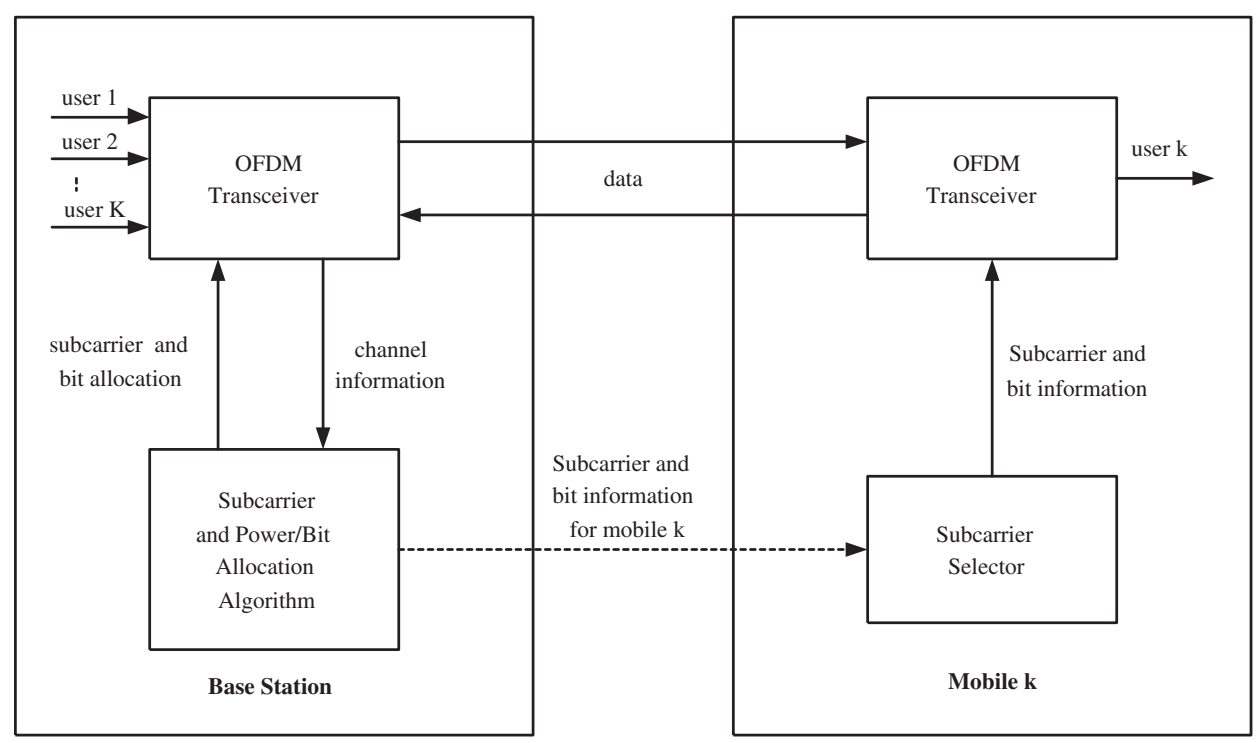

Fig. 1. Multiuser OFDM System Block Diagram

channel. The above two assumptions are not practical in real wireless environments. Effective methods to relax these assumptions are under investigation.

Ideally, subcarriers and power should be allocated jointly to achieve the optimal solution. However, this poses an extremely heavy computational burden at the base station in order to reach an optimal allocation. Furthermore, the base station has to rapidly compute the optimal subcarrier and power allocation if the wireless channel changes quickly. Hence suboptimal algorithms with lower complexity are preferred for cost-effective implementations. Separating the subcarrier and power allocation is a way to reduce the complexity since the number of variables in the objective function is almost reduced by half. In this paper, subcarrier allocation is assumed to be performed by the algorithm in [7].

Since the subcarrier allocation is performed before the power allocation, the optimization problem considered in this paper is formulated as

$$
\max _{p_{k, n}} \sum_{k=1}^{K} \sum_{n \in \Omega_{k}} \frac{1}{N} \log _{2}\left(1+\frac{p_{k, n} h_{k, n}^{2}}{N_{0} \frac{B}{N}}\right)
$$

$$
\begin{aligned}
\text { subject to: } & \sum_{k=1}^{K} \sum_{n \in \Omega_{k}} p_{k, n} \leq P_{\text {total }} \\
& p_{k, n} \geq 0 \text { for all } k, n \\
& \Omega_{k} \text { are disjoint for all } k \\
& \Omega_{1} \cup \Omega_{2} \cup \ldots \cup \Omega_{K} \subseteq\{1,2, \ldots, N\} \\
& R_{1}: R_{2}: \ldots: R_{K}=\gamma_{1}: \gamma_{2}: \ldots: \gamma_{K}
\end{aligned}
$$

where $K$ is the total number of users; $N$ is the total number of subcarriers; $N_{0}$ is the power spectrum density of additive white Gaussian noise; $B$ and $P_{\text {total }}$ are the overall available bandwidth and power, respectively; $p_{k, n}$ is the power allocation for user $k$ in the subcarrier $n ; h_{k, n}$ is the channel gain for user $k$ in subcarrier $n ; \Omega_{k}$ is the set of subcarriers for user $k$ and they are mutually exclusive; $N_{k}$ is the number of subcarriers in $\Omega_{k} ; R_{k}$ is the channel capacity for user $k$ defined as

$$
R_{k}=\sum_{n \in \Omega_{k}} \frac{1}{N} \log _{2}\left(1+\frac{p_{k, n} h_{k, n}^{2}}{N_{0} \frac{B}{N}}\right)
$$

and $\left\{\gamma_{i}\right\}_{i=1}^{K}$ is a set of predetermined values that are used to ensure proportional fairness among users. When all $\gamma_{i}$ 's are the same value, the objective function in (1) is similar to the objective function for the rate adaptive method [7], because maximizing the overall capacity while making all $R_{k}$ 's equal is equivalent to maximizing the minimum user's capacity. Furthermore, the last constraint in (1) also prevents the situation that no resource allocation scheme exists because of impractical capacity requirements from some of the users.

\section{Optimal Power Allocation}

We define $H_{k, n}=\frac{h_{k, n}^{2}}{N_{0} \frac{B}{N}}$ as the channel-to-noise gain for user $k$ in subcarrier $n$. The optimization problem in (1) is equivalent to finding the maximum of the following cost function:

$$
\begin{aligned}
L= & \sum_{k=1}^{k=K} \sum_{n \in \Omega_{k}} \frac{1}{N} \log _{2}\left(1+p_{k, n} H_{k, n}\right) \\
& +\lambda_{1}\left(\sum_{k=1}^{K} \sum_{n \in \Omega_{k}} p_{k, n}-P_{\text {total }}\right) \\
& +\sum_{k=2}^{K} \lambda_{k}\left(\sum_{n \in \Omega_{1}} \frac{1}{N} \log _{2}\left(1+p_{1, n} H_{1, n}\right)\right. \\
& \left.-\frac{\gamma_{1}}{\gamma_{k}} \sum_{n \in \Omega_{k}} \frac{1}{N} \log _{2}\left(1+p_{k, n} H_{k, n}\right)\right)
\end{aligned}
$$


We differentiate (3) with respect to $p_{k, n}$ and set each derivative to 0 to obtain

$$
\begin{aligned}
\frac{\partial L}{\partial p_{1, n}}= & \frac{1}{N \ln 2} \frac{H_{1, n}}{1+H_{1, n} p_{1, n}} \\
& +\lambda_{1}+\sum_{k=2}^{K} \lambda_{k} \frac{1}{N \ln 2} \frac{H_{1, n}}{1+H_{1, n} p_{1, n}}=0 \\
\frac{\partial L}{\partial p_{k, n}}= & \frac{1}{N \ln 2} \frac{H_{k, n}}{1+H_{k, n} p_{k, n}} \\
& +\lambda_{1}-\lambda_{k} \frac{\gamma_{1}}{\gamma_{k}} \frac{1}{N \ln 2} \frac{H_{k, n}}{1+H_{k, n} p_{k, n}}=0
\end{aligned}
$$

for $k=2,3, \ldots, K$ and $n \in \Omega_{k}$.

\section{A. Power Distribution for a Single User}

In this section, the optimal power distribution strategy for a single user is derived.

From either (4) or (5), we may obtain

$$
\frac{H_{k, m}}{1+H_{k, m} p_{k, m}}=\frac{H_{k, n}}{1+H_{k, n} p_{k, n}}
$$

for $m, n \in \Omega_{k}$ and $k=1,2, \ldots, K$. Without loss of generality, it could be assumed that $H_{k, 1} \leq H_{k, 2} \leq \ldots \leq H_{k, N_{k}}$ for $k=1,2, \ldots, K$ and $N_{k}$ is number of subcarriers in $\Omega_{k}$. Thus, (6) can be rewritten as

$$
p_{k, n}=p_{k, 1}+\frac{H_{k, n}-H_{k, 1}}{H_{k, n} H_{k, 1}}
$$

for $n=1,2, \ldots, N_{k}$ and $k=1,2, \ldots, K$.

Equation (6) shows the optimal power distribution for a single user. More power will be put into the subcarriers with high channel-to-noise gain. This is water-filling in the frequency domain.

By defining $P_{k, t o t}$ as the total power allocated for user $k$, with (7) $P_{k, t o t}$ can be expressed as

$$
P_{k, t o t}=\sum_{n=1}^{N_{k}} p_{k, n}=N_{k} p_{k, 1}+\sum_{n=2}^{N_{k}} \frac{H_{k, n}-H_{k, 1}}{H_{k, n} H_{k, 1}}
$$

for $k=1,2, \ldots, K$.

\section{B. Power Distribution among Users}

Once the set $\left\{P_{k, t o t}\right\}_{k=1}^{K}$ is known, the power allocation scheme can be determined by (7) and (8). In this section, power allocation among users will be discussed in order to compute the set $\left\{P_{k, t o t}\right\}_{k=1}^{K}$.

Specifically, the total power constraint and capacity ratio constraints in (1) are used to obtain $\left\{P_{k, t o t}\right\}_{k=1}^{K}$. With (6) and (8), the capacity ratio constraints can be expressed as

$$
\begin{aligned}
& \frac{1}{\gamma_{1}} \cdot \frac{N_{1}}{N}\left(\log _{2}\left(1+H_{1,1} \frac{P_{1, t o t}-V_{1}}{N_{1}}\right)+\log _{2} W_{1}\right) \\
= & \frac{1}{\gamma_{k}} \cdot \frac{N_{k}}{N}\left(\log _{2}\left(1+H_{k, 1} \frac{P_{k, t o t}-V_{k}}{N_{k}}\right)+\log _{2} W_{k}\right)
\end{aligned}
$$

for $k=2,3, \ldots, K$ and $V_{k}, W_{k}$ are defined as

$$
V_{k}=\sum_{n=2}^{N_{k}} \frac{H_{k, n}-H_{k, 1}}{H_{k, n} H_{k, 1}}
$$

and

$$
W_{k}=\left(\prod_{n=2}^{N_{k}} \frac{H_{k, n}}{H_{k, 1}}\right)^{\frac{1}{N_{k}}}
$$

for $k=1,2, \ldots, K$

Adding the total power constraints

$$
\sum_{k=1}^{K} P_{k, t o t}=P_{t o t a l}
$$

there are $K$ variables $\left\{P_{k, t o t}\right\}_{k=1}^{K}$ in the set of $K$ equations in (9) and (12). Solving the set of functions will provide the optimal power allocation scheme. The set of equations is, in general, nonlinear. Iterative methods, such as Newton-Raphson method [14] or Quasi-Newton method [14], can be used to obtain the solution, with a certain amount of computational effort. In the Newton-Raphson method, the computational complexity primarily comes from finding the update direction. The Jacobian matrix of the functions has nonzero elements only on the first row, the first column and the main diagonal. Thus the computational complexity of each iteration can be shown to be $\mathcal{O}(K)$. However, under certain conditions, lower computationally complex algorithms can be applied to find the optimal or near-optimal solution. Two special cases are analyzed below.

1) Linear Case: If $N_{1}: N_{2}: \ldots: N_{K}=\gamma_{1}: \gamma_{2}: \ldots: \gamma_{K}$, then the set of equations can be transformed into a set of linear equations with the following expression

$$
\left[\begin{array}{cccc}
1 & 1 & \ldots & 1 \\
1 & a_{2,2} & \ldots & 0 \\
\vdots & \vdots & \ddots & \vdots \\
1 & 0 & \ldots & a_{K, K}
\end{array}\right]\left[\begin{array}{c}
P_{1, t o t} \\
P_{2, t o t} \\
\vdots \\
P_{K, t o t}
\end{array}\right]=\left[\begin{array}{c}
P_{t o t a l} \\
b_{2} \\
\vdots \\
b_{K}
\end{array}\right]
$$

where

$$
a_{k, k}=-\frac{N_{1}}{N_{k}} \frac{H_{k, 1} W_{k}}{H_{1,1} W_{1}}
$$

and

$$
b_{k}=\frac{N_{1}}{H_{1,1} W_{1}}\left(W_{k}-W_{1}+\frac{H_{1,1} V_{1} W_{1}}{N 1}-\frac{H_{k, 1} V_{k} W_{k}}{N_{k}}\right)
$$

for $k=2,3, \ldots, K$. By substitution, the solution to (13) can be obtained with a computational complexity of $\mathcal{O}(K)$.

2) High Channel-to-Noise Ratio: In adaptive modulation, the linear condition rarely happens and the set of equations remains nonlinear, which requires considerably more computation to solve. However, if the channel-to-noise ratio is high, approximations can be made to simplify the problem.

First consider (10), in which $V_{k}$ could be relatively small compared to $P_{k, t o t}$ if the channel-to-noise ratios are high. Furthermore, if adaptive subcarrier allocation is used, the best 
subcarriers will be chosen and they have relatively small channel gain differences among them. Thus, the first approximation is $V_{k}=0$.

Second, assuming that the base station can provide a large amount of power and the high channel-to-noise ratio assumption holds, the term $H_{k, 1} P_{k, t o t} / N_{k}$ is much larger than 1 .

With the above two approximations, (9) can be simplified as

$$
\left(\frac{H_{1,1} W_{1}}{N_{1}}\right)^{\frac{N_{1}}{\gamma_{1}}}\left(P_{1, t o t}\right)^{\frac{N_{1}}{\gamma_{1}}}=\left(\frac{H_{k, 1} W_{k}}{N_{k}}\right)^{\frac{N_{k}}{\gamma_{k}}}\left(P_{k, t o t}\right)^{\frac{N_{k}}{\gamma_{k}}}
$$

where $k=2,3, \ldots, K$.

Substituting (16) into (12), a single equation in the variable $P_{1, t o t}$ could be derived as

$$
F\left(P_{1, t o t}\right)=\sum_{k=1}^{K} c_{k}\left(P_{1, t o t}\right)^{d_{k}}-P_{\text {total }}=0
$$

where

$$
c_{k}= \begin{cases}1 & \text { if } k=1 \\ \frac{N_{k}}{H_{k, 1} W_{k}} \cdot\left(\frac{H_{1,1} W_{1}}{N_{1}}\right)^{\frac{N_{1} \gamma_{k}}{N_{k} \gamma_{1}}} & \text { if } k=2,3, \ldots, K\end{cases}
$$

and

$$
d_{k}= \begin{cases}1 & \text { if } k=1 \\ \frac{N_{1} \gamma_{k}}{N_{k} \gamma_{1}} & \text { if } k=2,3, \ldots, K\end{cases}
$$

Numerical algorithms, such as Newton's root-finding method [13] or the false position method [13], could be used to find the zero of (17). For each iteration of these numerical algorithms, Newton's method requires $2 K$ multiplications, $2 K$ power operations, and 1 division to find an update, while the false position method needs $K+1$ multiplications, $K$ power operations, and 1 division. In both cases, the operations of addition are not taken into account.

\section{Existence of Solutions}

1) Solution to Single User Power Allocation: For a certain user $k$, there is no power allocation if $V_{k}>P_{k, t o t}$. This situation could happen when a subcarrier is allocated to a user who does not have a high channel gain in that subcarrier. The greedy water-filling algorithm would rather stop using this subcarrier. In case this situation happens, the set of $\Omega_{k}$, as well as the corresponding values of $N_{k}, V_{k}$ and $W_{k}$, would need to be updated and the power allocation algorithm presented in this paper should be executed again.

2) Solution to Multiuser Power Allocation: In case that the channel-to-noise ratio is high, there is one and only one solution to (17) since every term in the summation monotonically increases and (17) has different signs at $P_{1, t o t}=0$ and $P_{1, t o t}=P_{\text {total }}$. A numerical algorithm can be used to find the solution to (17). The complexity of finding the solution will primarily rely on the choice of the numerical algorithm and the precision required in the results. After $P_{1, t o t}$ is found, $\left\{P_{k, t o t}\right\}_{k=2}^{K}$ can be calculated using (16). Then the overall power allocation scheme can be determined with (7) and (8).
In general, it can be proved that there must be an optimal subcarrier and power allocation scheme that satisfies the proportional fairness constraints and the total power constraint. Furthermore, the optimal scheme must utilize all available power. Several facts lead to the above conclusion. First, to a certain user, that capacity of the user is maximized if waterfilling algorithm is adopted. Furthermore, the capacity function is continuous with respect to the total available power to that user. In other words, $R_{k}\left(P_{k, t o t}\right)$ is continuous with $P_{k, t o t}$. Second, if the optimal allocation scheme does not use all the available power, there is always a way to redistribute the unused power among users while maintaining the capacity ratio constraints, since $R_{k}\left(P_{k, t o t}\right)$ is continuous with $P_{k, t o t}$ for all $k$. Thus, the overall capacity is further increased.

\section{Simulation Results}

In this section, simulation results are presented. Let $\gamma_{1}$ : $\gamma_{2}: \ldots: \gamma_{K}=1: 1: \ldots: 1$ and the objective of the optimization problem (1) turns out to maximize the minimum user's capacity as in [7], since the minimum user's capacity is maximized when all the users have the same capacity and the overall capacity is maximized. Thus in the simulations presented here, the minimum user's capacity is compared. In [7], a suboptimal algorithm is proposed to achieve near optimal capacity using adaptive subcarrier allocation. However, the suboptimal algorithm in [7] assumes that equal power is distributed into every subcarrier. When the number of users increases, equal power distribution does not equalize every user's capacity. By transferring power from the users with high capacity to the users with low capacity, the minimum user's capacity could be even increased. In the simulations presented here, we use the suboptimal algorithm in [7] to allocate the subcarriers and then apply the optimal power allocation scheme proposed in this paper. Both of these adaptive schemes are compared with the fixed time division multiple access (TDMA) resource allocation scheme. Here, we represent the suboptimal algorithm in [7] as follows:

1) Initialization

set $R_{k}=0, \Omega_{k}=\varnothing$ for $k=1,2, \ldots, K$ and $A=$ $\{1,2, \ldots, N\}$

2) for $k=1$ to $K$

a) find $n$ satisfying $\left|H_{k, n}\right| \geq\left|H_{k, j}\right|$ for all $j \in A$

b) let $\Omega_{k}=\Omega_{k} \cup\{n\}, A=A-\{n\}$ and update $R_{k}$ according to (2)

3) while $A \neq \varnothing$

a) find $k$ satisfying $R_{k} \leq R_{i}$ for all $i, 0 \leq i \leq K$

b) for the found $k$, find $n$ satisfying $\left|H_{k, n}\right| \geq\left|H_{k, j}\right|$ for all $j \in A$

c) for the found $k$ and $n$, let $\Omega_{k}=\Omega_{k} \cup\{n\}, A=$ $A-\{n\}$ and update $R_{k}$ according to (2)

In all the simulations, the wireless channel is modeled as a frequency-selective multipath channel consisting of six independent Rayleigh multipaths, with an exponentially decaying profile. The maximum delay spread is $5 \mu \mathrm{s}$. The maximum Doppler frequency spread is $30 \mathrm{~Hz}$. Channel information is 


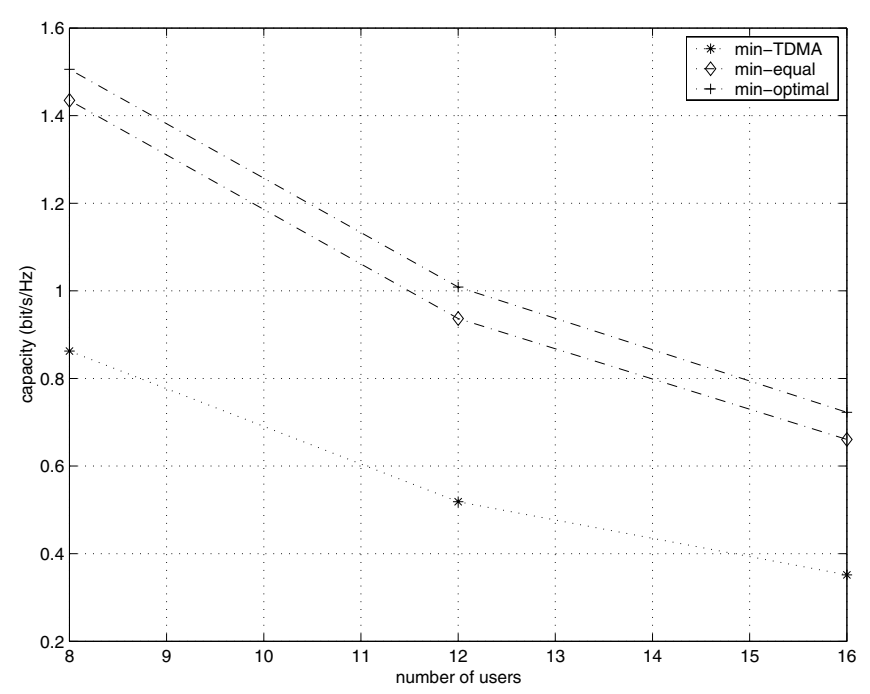

Fig. 2. Capacity in multiuser OFDM vs. user number.

sampled every $0.5 \mathrm{~ms}$ to update the subchannel and power allocation. The total power available at the base station is 64 W. The power spectrum density of additive white Gaussian noise is $-80 \mathrm{dBW} / \mathrm{Hz}$. The overall bandwidth is $1 \mathrm{MHz}$ and it is divided into 64 subcarriers. The maximum path loss difference is $40 \mathrm{~dB}$. The user locations are assumed to be equally distributed.

Fig. 2 shows the capacity vs. the user number in the OFDM system. We can see from Fig. 2 that adaptive resource allocations can achieve significant capacity gain over nonadaptive TDMA. Also the adaptive allocation scheme with optimal power allocation will achieve even higher capacity than the scheme with power equally distributed.

Fig. 3 shows the capacity gain vs. the user number. From Fig. 3, we can see the more users in the system, the higher the capacity gain adaptive schemes can achieve. This could be explained by multiuser diversity. The more users in the system, the lower the probability that a subcarrier is in deep fading to all the users. Also we can see that in a system of 16 users, the adaptive scheme with the proposed power allocation achieves $17 \%$ more capacity gain than the scheme with equal power, when compared to fixed TDMA.

\section{CONClusion}

In this paper, a proportional rate adaptive optimization in MU-OFDM is analyzed. Subcarrier and power allocation are carried out separately. The optimal power allocation to a determined subcarrier scheme is developed. Arbitrary proportional fairness can be achieved with the proposed algorithm. Simulation results shows that the proposed optimal power allocation improves the capacity significantly over fixed TDMA, and outperforms previously derived suboptimal power distribution schemes. In general, a set of nonlinear equations has to be solved to find the optimal power allocation. However, by taking advantage of the structure of the equations, the complexity of the proposed method is shown to be $\mathcal{O}(K)$.

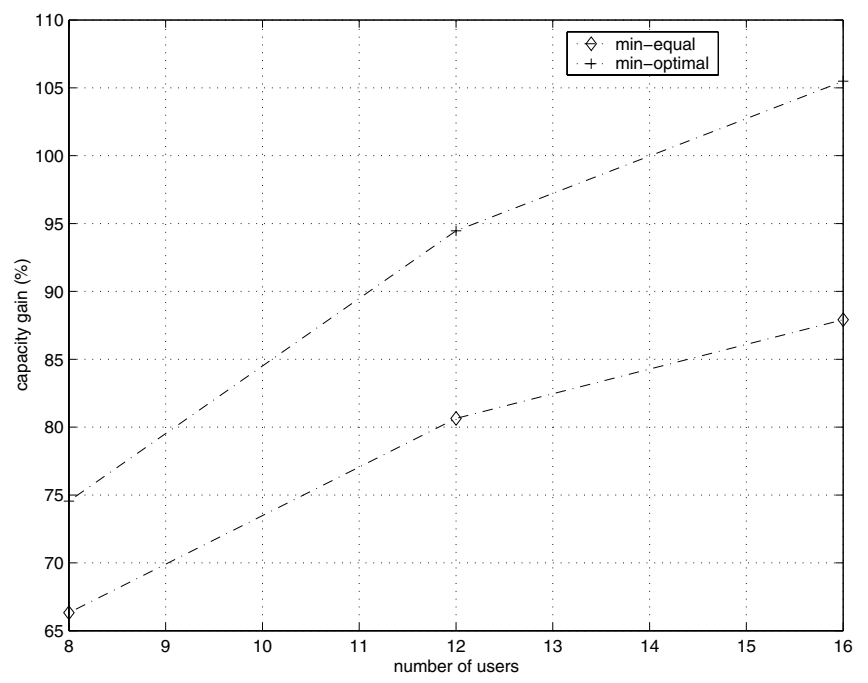

Fig. 3. Capacity gain over TDMA vs. user number, using curves in Fig. 2

Two special cases with even lower computational complexity are also discussed.

\section{REFERENCES}

[1] H. Sampath, S. Talwar, J. Tellado, V. Erceg, and A. Paulraj, "A Fourthgeneration MIMO-OFDM Broadband Wireless System: Design, Performance, and Field Trial Results," IEEE Communications Magazine, vol. 40, no. 9, pp. 143-149, Sep. 2002.

[2] T. S. Rappaport, A. Annamalai, R. M. Buehrer, and W. H. Tranter, "Wireless Communications: Past Events and a Future Perspective," IEEE Communications Magazine, vol. 40, no. 5, pp. 148-161, May 2002.

[3] J. A. C. Bingham, "Multicarrier Modulation for Data Transmisson: an Idea whose Time Has Come," IEEE Communications Magazine, vol. 28, no. 5, pp. 5-14, May 1990.

[4] E. Lawrey, "Multiuser OFDM," Proc. IEEE International Symposium on Signal Processing and its Applications, vol. 2, pp. 761-764, Aug. 1999.

[5] C. Y. Wong, R. S. Cheng, K. B. Letaief, and R. D. Murch, "Multicarrier OFDM with Adaptive Subcarrier, Bit, and Power Allocation," IEEE Journal on Selected Areas in Communications, vol. 17, no. 10, Oct. 1999.

[6] J. Jang and K. B. Lee, "Transmit Power Adaptation for Multiuser OFDM Systems", IEEE Journal on Selected Areas in Communications, vol. 21, no. 2, pp. 171-178, Feb. 2003.

[7] W. Rhee and J. M. Cioffi, "Increasing in Capacity of Multiuser OFDM System Using Dynamic Subchannel Allocation," in Proc. IEEE International Vehicular Technology Conference, vol. 2, pp. 1085-1089, May 2000.

[8] I. Kim, H. L. Lee, B. Kim, and Y. H. Lee, "On the Use of Linear Programming for Dynamic Subchannel and Bit Allocation in Multiuser OFDM," in Proc. IEEE Global Communications Conference, vol. 6, pp. 3648-3652, Nov. 2001.

[9] N. Jindal and A. Goldsmith, "Capacity and Optimal Power Allocation for Fading Broadcast Channels with Minimum Rates," in Proc. IEEE Global Communications Conference, vol. 2, pp. 1292-1296, Nov. 2001.

[10] M. C. Hool, J. Tellado, and J. M. Cioffi, "Dual QoS Loading Algorithms for DMT Systems Offering CBR and VBR Services," in Proc. IEEE Global Communications Conference, vol. 1, pp. 25-30, Nov. 1998.

[11] T. S. Rappaport, Wireless Communications: Principles and Practice, Prentice Hall PTR, 2002.

[12] T. M. Cover and J. A. Thomas, Elements of Information Theory, John Wiley \& Sons, Inc. 1991.

[13] M. Abramowitz and I. A. Stegun, Handbook of Mathematical Functions with Formulas, Graphs, and Mathematical Tables, 9th printing. New York: Dover, 1972.

[14] R. Baldick, Optimization of Engineering Systems Course Notes, The University of Texas at Austin, http://www.ece.utexas.edu/baldick/. 